\title{
Mode-coupling and the pygmy dipole resonance in a relativistic two-phonon model
}

\author{
Elena Litvinova, ${ }^{1,2,3}$ Peter Ring, ${ }^{4}$ and Victor Tselyaev $^{5}$ \\ ${ }^{1}$ GSI Helmholtzzentrum für Schwerionenforschung, 64291 Darmstadt, Germany \\ ${ }^{2}$ Institut für Theoretische Physik, Goethe-Universität, 60438 Frankfurt am Main, Germany \\ ${ }^{3}$ Institute of Physics and Power Engineering, 249033 Obninsk, Russia \\ ${ }_{4}$ Physik-Department der Technischen Universität München, D-85748 Garching, Germany \\ ${ }^{5}$ Nuclear Physics Department, St. Petersburg State University, 198504 St. Petersburg, Russia
}

(Dated: July 17, 2018)

A two-phonon version of the relativistic quasiparticle time blocking approximation (RQTBA-2) represents a new class of many-body models for nuclear structure calculations based on the covariant energy density functional. As a fully consistent extension of the relativistic quasiparticle random phase approximation (RQRPA), the two-phonon RQTBA implies a fragmentation of nuclear states over two-quasiparticle and two-phonon configurations. This leads, in particular, to a splitting-out of the lowest $1^{-}$state as a member of the two-phonon $\left[2^{+} \otimes 3^{-}\right]$quintuplet from the RQRPA pygmy dipole mode, thus establishing a physical mixing between these three modes. The inclusion of the two-phonon configurations in the model space allows to describe the positions and the reduced transition probabilities of the lowest $1^{-}$states in isotopes ${ }^{116,120} \mathrm{Sn}$ as well as the low-energy fraction of the dipole strength without any adjustment procedures. The model is also applied to the low-lying dipole strength in neutron-rich ${ }^{68,70,72} \mathrm{Ni}$ isotopes. Recent experimental data for ${ }^{68} \mathrm{Ni}$ are reproduced fairly well.

The theoretical description of nuclear low-lying dipole strength remains among the most important problems in nuclear structure and nuclear astrophysics [1]. Measurements of the dipole strength by means of high resolution nuclear resonance fluorescence [2, 3, 4, 5, 6, 6] resolve the fine structure of the spectra below the neutron threshold. Unique spectroscopic information about neutron-rich medium-mass and heavy nuclei have been obtained in recent experiments with Coulomb dissociation [8, 9] and virtual photon scattering [10]. This offers 
exciting opportunities for microscopic nuclear structure models to describe the fine structure of the dipole spectra below the neutron threshold and to help in analyzing the experimental data.

Measurements of the low-lying dipole strength above and below the neutron threshold are performed with different nuclear reactions, which have reduced sensitivity in the area around the threshold. Therefore, a correct comparison of the calculated pygmy strength with the data is still problematic. The collectivity of the pygmy mode is another subject of discussions. Self-consistent relativistic QRPA calculations [11] produce a highly collective pygmy mode, in contrast to the results of the non-relativistic approaches [12, 13]. However, in Ref. [14] it is pointed out that the collectivity of the pygmy mode is restored within non-relativistic QRPA calculations with Skyrme forces when a fully self-consistent scheme is employed.

Since the pygmy dipole mode has essentially surface nature, it mixes with other surface modes, especially with low-lying ones. This supposition has been confirmed by explicit RQTBA calculations [15, 16] for tin and nickel isotopes and $\mathrm{N}=50$ isotones. It has been found that the pygmy mode, arising in RQRPA as a single state or as very few low lying dipole states with isoscalar character, is strongly fragmented over many states in a broad energy region due to the coupling to phonons. As a result, some fraction of the strength is located well below the original position of the RQRPA pygmy mode. Compared to existing data, the RQTBA describes the total fraction of the dipole strength below the neutron threshold very reasonably [15, 16]. However, in order to account for the fine structure of the spectrum, more correlations should be included in the microscopic model. In particular, it has to reproduce the lowest dipole state in vibrational nuclei, which is identified as a member of the quintuplet $2_{1}^{+} \otimes 3_{1}^{-}$, as it was predicted in Refs. [17, 18] and observed in spectra of spherical nuclei [19, 20, 21, 22].

In the present work, the two-phonon version of the quasiparticle time blocking approximation (QTBA) proposed in Ref. [23] is employed to introduce correlations between the two quasiparticles within the $2 \mathrm{q} \otimes$ phonon configurations of the RQTBA. Therefore, a fragmentation of the nuclear states over the two-phonon configurations appears in the excitation spectra in addition to the spreading over the two-quasiparticle states. As well as in the conventional RQTBA, excitations in Fermi-systems with even particle number are described by the Bethe-Salpeter equation (BSE) containing both static and energy-dependent residual in- 

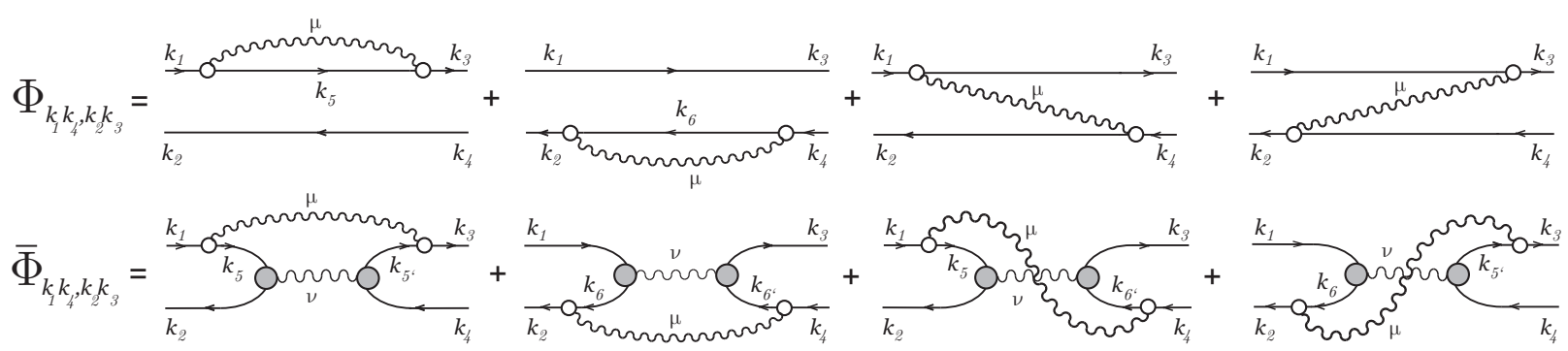

FIG. 1: The correspondence between the $2 \mathrm{q} \otimes$ phonon amplitude $\Phi$ of the conventional phonon coupling model and the two-phonon amplitude $\bar{\Phi}$ of the two-phonon model in a diagrammatic representation. Solid lines with arrows and latin indices denote the single-quasiparticle nucleonic propagators, wavy curves with greek indices the phonon propagators, empty circles represent phonon vertices, and grey circles together with the two nucleonic lines denote the RQRPA transition densities (see text).

teractions. This equation for the nuclear response function $R(\omega)$ in the doubled quasiparticle space reads:

$$
R_{k_{1} k_{4}, k_{2} k_{3}}^{\eta \eta^{\prime}}(\omega)=\tilde{R}_{k_{1} k_{2}}^{(0) \eta}(\omega) \delta_{k_{1} k_{3}} \delta_{k_{2} k_{4}} \delta^{\eta \eta^{\prime}}+\tilde{R}_{k_{1} k_{2}}^{(0) \eta}(\omega) \sum_{k_{5} k_{6} \eta^{\prime \prime}} \bar{W}_{k_{1} k_{6}, k_{2} k_{5}}^{\eta \eta^{\prime \prime}}(\omega) R_{k_{5} k_{4}, k_{6} k_{3}}^{\eta^{\prime \prime} \eta^{\prime}}(\omega)
$$

where the indices $k_{i}$ run over single-particle quantum numbers including states in the Dirac sea and the indices $\eta, \eta^{\prime}, \eta^{\prime \prime}$ numerate forward $(+)$ and backward $(-)$ components in the doubled quasiparticle space. The quantity $\tilde{R}_{k_{1} k_{2}}^{(0) \eta}(\omega)=1 /\left(\eta \omega-E_{k_{1}}-E_{k_{2}}\right)$ describes the free propagation of two quasiparticles with their Bogoliubov's energies $E_{k_{1}}$ and $E_{k_{2}}$ in the mean field between subsequent interactions with the amplitude

$$
\bar{W}_{k_{1} k_{4}, k_{2} k_{3}}^{\eta \eta^{\prime}}(\omega)=\tilde{V}_{k_{1} k_{4}, k_{2} k_{3}}^{\eta \eta^{\prime}}+\left[\Phi_{k_{1} k_{4}, k_{2} k_{3}}^{\eta}(\omega)-\Phi_{k_{1} k_{4}, k_{2} k_{3}}^{\eta}(0)\right] \delta^{\eta \eta^{\prime}}
$$

Here $\tilde{V}$ is the static part of the effective residual quasiparticle interaction. In the present work, as in Refs. [15, 16], it is derived from the covariant energy density functional (CEDF) with the parameter set NL3 [24] as a one-meson exchange interaction with a non-linear self-coupling between the mesons. Pairing correlations are introduced into the relativistic energy functional as an independently parameterized term. In the $2 \mathrm{q} \otimes$ phonon version of the RQTBA the energy-dependent residual interaction $\Phi(\omega)$ is derived from the energydependent self-energy by the consistency condition and calculated within the quasiparticle time blocking approximation. This approximation means that, due to the time projection 
in the integral part of the BSE, the two-body propagation through states with a more complicated structure than $2 \mathrm{q} \otimes$ phonon is blocked [23].

In the present work we go a step further and consider a modified version of the RQTBA which includes additional correlations between two quasiparticles inside the $2 \mathrm{q} \otimes$ phonon configurations. This version of the model has been proposed in Ref. [23] for the non-relativistic case. It has been noticed that the energy-dependent resonant part of the two-quasiparticle amplitude $\Phi(\omega)$ can be factorized to extract the two-quasiparticle intermediate propagator with the frequency shifted by the phonon energy. In the relativistic RQTBA it takes the following form:

$$
\Phi_{k_{1} k_{4}, k_{2} k_{3}}^{\eta}(\omega)=\sum_{k_{5} k_{6}, \mu} \zeta_{k_{1} k_{2} ; k_{5} k_{6}}^{\mu \eta} \tilde{R}_{k_{5} k_{6}}^{(0) \eta}\left(\omega-\eta \Omega_{\mu}\right) \zeta_{k_{3} k_{4} ; k_{5} k_{6}}^{\mu \eta *}
$$

where $\tilde{R}_{k_{5} k_{6}}^{(0) \eta}\left(\omega-\eta \Omega_{\mu}\right)$ are the matrix elements of the two-quasiparticle propagator in the mean field with the frequency shifted forward or backward by the phonon energy $\Omega_{\mu}$,

$$
\zeta_{k_{1} k_{2} ; k_{5} k_{6}}^{\mu(+)}=\delta_{k_{1} k_{5}} \gamma_{\mu ; k_{6} k_{2}}^{(-)}-\gamma_{\mu ; k_{1} k_{5}}^{(+)} \delta_{k_{6} k_{2}}=-\zeta_{k_{2} k_{1} ; k_{6} k_{5}}^{\mu(-) *}
$$

and $\gamma_{\mu ; k_{1} k_{2}}^{\eta}$ are the quasiparticle-phonon coupling vertices defined in Ref. [15]. In the graphic expression of the amplitude (3) in the upper line of the Fig. 1 the propagator $\tilde{R}_{k_{1} k_{2}}^{(0) \eta}$ is represented by the two straight nucleonic lines between the circles denoting emission and absorption of the phonon by a single quasiparticle with amplitudes $\gamma_{\mu ; k_{1} k_{2}}^{\eta}$. The extension of this model proposed in Ref. [23] consists in the following: we introduce RQRPA correlations into the intermediate two-quasiparticle propagator so that the two-phonon configurations appear in the amplitude $\Phi(\omega)$ as it is shown in the lower line of the Fig. 1. The analytic expression of the new amplitude reads:

$$
\bar{\Phi}_{k_{1} k_{4}, k_{2} k_{3}}^{\eta}(\omega)=\frac{1}{2} \sum_{\mu, \nu} \frac{\bar{\zeta}_{\mu \nu ; k_{1} k_{2}}^{\eta} \bar{\zeta}_{\mu \nu ; k_{3} k_{4}}^{\eta *}}{\eta \omega-\Omega_{\mu}-\Omega_{\nu}}
$$

where

$$
\bar{\zeta}_{\mu \nu ; k_{1} k_{2}}^{(+)}=\sum_{k_{6}} \mathcal{R}_{\nu ; k_{1} k_{6}}^{(+)} \gamma_{\mu ; k_{6} k_{2}}^{(-)}-\sum_{k_{5}} \gamma_{\mu ; k_{1} k_{5}}^{(+)} \mathcal{R}_{\nu ; k_{5} k_{2}}^{(+)}=-\bar{\zeta}_{\mu \nu ; k_{2} k_{1}}^{(-) *}
$$

and $\mathcal{R}_{\nu ; k_{1} k_{2}}^{\eta}$ are the matrix elements of the RQRPA transition densities defined in Ref. [15] and corresponding to grey circles together with two nucleonic lines in Fig. 1. One can show that in the limit of the vanishing static interaction $\tilde{V}$ between the two intermediate quasiparticles Eq. (5) transforms to the Eq. (3) of the original (R)QTBA. As in conventional 
(R)QTBA, the elimination of double counting effects in the phonon coupling is performed by the subtraction of the static contribution of the amplitude $\bar{\Phi}$ from the residual interaction in Eq. (21), since the parameters of the CEDF have been adjusted to experimental data for ground states and include therefore already essential phonon contributions to the ground state. Therefore, the BSE in the two-phonon model has the same form as Eq. (1), but contains the amplitude $\bar{\Phi}$ instead of $\Phi$. In order to take the quasiparticle-phonon coupling into account in a consistent way, we have first calculated the vertices $\gamma^{\eta}$ of this coupling and the transition densities $\mathcal{R}^{\eta}$ within the self-consistent RQRPA using the static residual interaction $\tilde{V}$, as described in Ref. [15]. On the next step the BSE for the correlated propagator $R^{(e)}(\omega)$

$$
R^{(e)}(\omega)=\tilde{R}^{(0)}(\omega)+\tilde{R}^{(0)}(\omega)[\bar{\Phi}(\omega)-\bar{\Phi}(0)] R^{(e)}(\omega),
$$

has been solved in the Dirac-Hartree-BCS basis. Then, the BSE for the full response function $R(\omega)$

$$
R(\omega)=R^{(e)}(\omega)+R^{(e)}(\omega) \tilde{V} R(\omega)
$$

has been solved in the momentum-channel representations. The details are given in Ref. [15]. To describe the observed spectrum of a nucleus excited by a weak external field as, for instance, an electromagnetic field $P$, the microscopic strength function $S(E)$ is computed as:

$$
S(E)=-\frac{1}{2 \pi} \lim _{\Delta \rightarrow+0} \operatorname{Im}\left[\operatorname{Tr}\left(P^{\dagger} R(E+i \Delta) P\right)\right] .
$$

To illustrate the effect of the additional static correlations on spectra of nuclear excitations, we consider the dipole response of tin and nickel isotopes in the area below the giant dipole resonance (GDR). Fig. 2 displays the dipole strength function in ${ }^{116,120} \mathrm{Sn}$ calculated within the conventional RQTBA [15] and the two-phonon RQTBA-2 presented here. The strength functions obtained in this way are compared with the original RQRPA strength function because both of them originate from RQRPA by different fragmentation mechanisms. The first observation is that the total strength $\sum B(E 1) \uparrow$ below the neutron threshold is reduced in the RQTBA-2. For example, for ${ }^{116} \mathrm{Sn}$ we have $0.20 \mathrm{e}^{2} \mathrm{fm}^{2}$ below 8 $\mathrm{MeV}$ which agrees with $0.204(25) \mathrm{e}^{2} \mathrm{fm}^{2}$ obtained in the experiment of Ref. [25]. For ${ }^{120} \mathrm{Sn}$, if we include the relatively strong state at $8.08 \mathrm{MeV}$ into the integration region, this quantity is $0.31 \mathrm{e}^{2} \mathrm{fm}^{2}$, in agreement with the results of the quasiparticle phonon model (QPM) of 

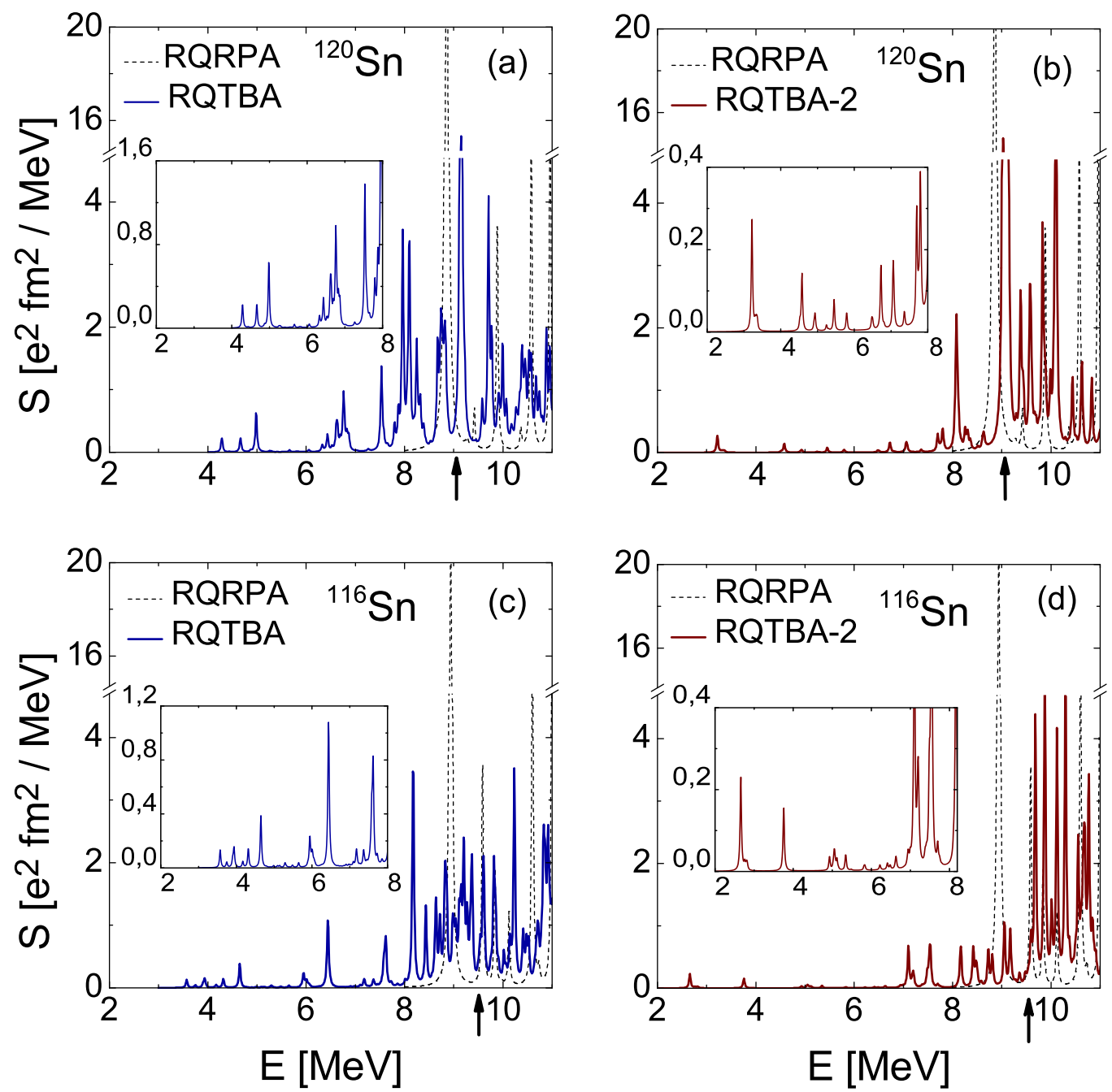

FIG. 2: Low-lying dipole spectra of ${ }^{116,120} \mathrm{Sn}$ calculated within the RQRPA (dashed curves), RQTBA (blue solid curve, panels (a,c)) and RQTBA-2 (red solid curve, panels (b,d)). A finite smearing parameter $\Delta=20 \mathrm{keV}$ has been used in the calculations. The inserts show the zoomed pictures of the spectra below $8 \mathrm{MeV}$. The arrows indicate the neutron thresholds.

$0.289 \mathrm{e}^{2} \mathrm{fm}^{2}[26]$. One can also notice that in both nuclei the major fraction of the RQRPA pygmy mode shown by the dashed curve is pushed up above the neutron threshold by the RQTBA-2 correlations.

The energies and the corresponding $B(E 1) \uparrow$ values of the lowest $1^{-}$states in the tin isotopes are listed in Table I. The experimental energies, $B(E 1) \uparrow$ values and the $R_{\omega}$ 
TABLE I: The energies, reduced transition probabilities and anharmonicities of the lowest $1^{-}$states in ${ }^{116,120} \mathrm{Sn}$ isotopes calculated in the relativistic two-phonon model, compared to data of Ref. [19], see text for details.

\begin{tabular}{ccccc}
\hline \hline & & $\begin{array}{c}\omega\left(1_{1}^{-}\right) \\
(\mathrm{MeV})\end{array}$ & $\begin{array}{c}\mathrm{B}(\mathrm{E} 1) \uparrow \\
\left(10^{-3} \mathrm{e}^{2} \mathrm{fm}^{2}\right)\end{array}$ & $R_{\omega}$ \\
\hline & RQTBA-2 & 2.66 & 14.5 & 0.94 \\
${ }^{116} \mathrm{Sn}$ & Exp. & 3.33 & $6.55(65)$ & 0.94 \\
\hline & RQTBA-2 & 3.22 & 16.9 & 0.95 \\
${ }^{120} \mathrm{Sn}$ & Exp. & 3.28 & $7.60(51)$ & 0.92 \\
\hline \hline
\end{tabular}

values, $R_{\omega}=\omega\left(1_{1}^{-}\right) /\left(\omega\left(2_{1}^{+}\right)+\omega\left(3_{1}^{-}\right)\right)$, are taken from Ref. [19]. Notice, however, that the measurements with the larger end point energies for the electron bremsstrahlung result in the larger $B(E 1) \uparrow$ values: $16.3(0.9)$ and $11.2(1.1)$ for ${ }^{116} \mathrm{Sn}$ and ${ }^{120} \mathrm{Sn}$, respectively [25, 27]. A good agreement with the data is obtained in spite of the fact that these tiny structures at about $3 \mathrm{MeV}$ originate by splitting-out from the very strong RQRPA pygmy states located at the neutron thresholds, that is described by the fully consistent inclusion of the two-phonon correlations without any adjustment procedures.

In the RQTBA-2 the position of the first $1^{-}$state is basically determined by the sum of the energies of the lowest $2^{+}$and $3^{-}$phonons. From the Eq. (15) one can see that the amplitude $\bar{\Phi}(\omega)$ consists of the pole terms with the poles at the energies which are sums of the two phonon energies. Therefore, the energy of the first $1^{-}$state is approximately equal to the sum of the energies of the lowest $2^{+}$and $3^{-}$phonons with some small correction introduced by the static residual interaction $\tilde{V}$, in agreement with data [19]. This correction is given by the quantity $R_{\omega}$, whose deviation from unity characterizes the two-phonon anharmonicity, see Table I. For example, in ${ }^{120} \mathrm{Sn}$ the energies of the $2_{1}^{+}$and $3_{1}^{-}$phonons calculated within the RQRPA are obtained at $1.48 \mathrm{MeV}$ and $1.90 \mathrm{MeV}$, respectively, explaining the position of the $1_{1}^{-}$state at $3.22 \mathrm{MeV}$ which is identified experimentally as a member of the $2_{1}^{+} \otimes 3_{1}^{-}$ quintuplet.

The electric dipole strengths in neutron rich ${ }^{68,70,72} \mathrm{Ni}$ isotopes are displayed in Fig. 3, For all three isotopes we found a redistribution of the low-lying strength as compared to the RQTBA calculations of Ref. [16]. The calculated strength distribution in ${ }^{68} \mathrm{Ni}$ has 

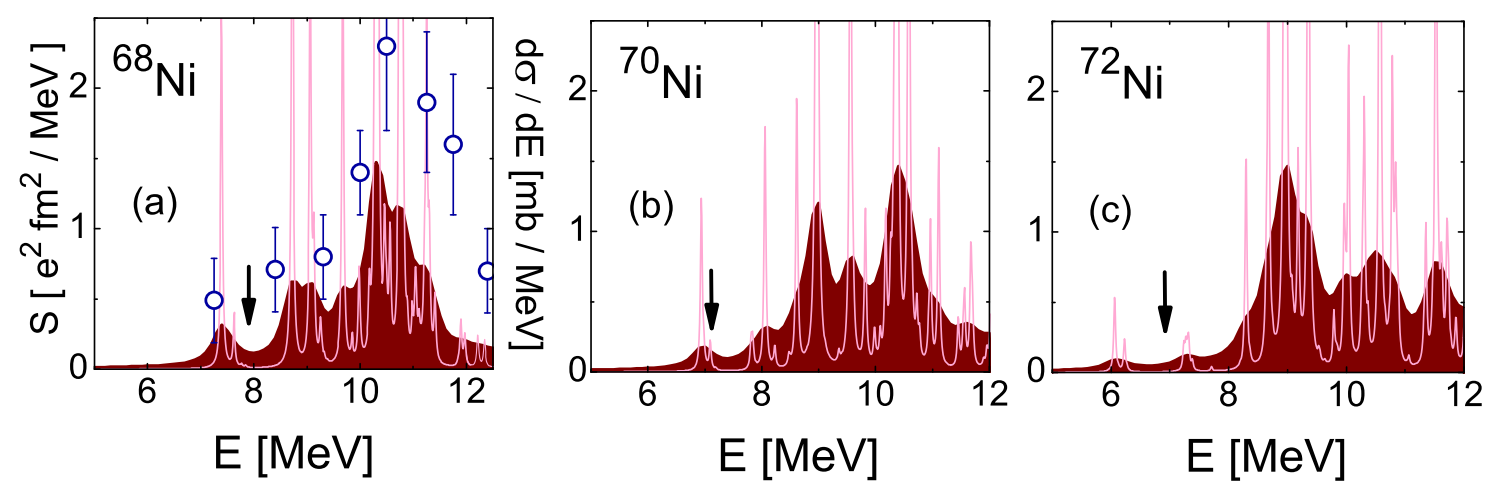

FIG. 3: Low-lying dipole spectra of ${ }^{68,70,72} \mathrm{Ni}$ calculated within the RQTBA-2 with a smearing of $20 \mathrm{keV}$ (thin curves, pink) and $200 \mathrm{keV}$ (filled area). Panel (a) contains also the data from Ref. [10] (open circles, units on the right). The arrows show the neutron thresholds.

its maximum at $10.30 \mathrm{MeV}$ and the total strength below $12 \mathrm{MeV}$ is $2.73 \mathrm{e}^{2} \mathrm{fm}^{2}$, while the corresponding fraction of the energy weighted sum rule (EWSR) is $7.8 \%$ of the total integrated photoabsorption cross section and $11 \%$ of the Thomas-Reiche-Kuhn sum rule. These characteristics are in agreement with the recent data of Ref. [10]. The RQTBA-2 dipole strength distributions presented in Fig. [3 for the ${ }^{70,72} \mathrm{Ni}$ isotopes can be suggested as predictions for possible future measurements in these nuclei.

In summary, the two-phonon version of the relativistic time blocking approximation is presented. Within this model it has been shown how the RQRPA modes are fragmented due to the coupling to two-phonon configurations, thus explaining the physical connection between the pygmy dipole mode and the $1^{-}$member of the $2_{1}^{+} \otimes 3_{1}^{-}$quintuplet. A very reasonable description of the lowest $1^{-}$states in ${ }^{116,120} \mathrm{Sn}$ has been achieved within the fully consistent scheme avoiding any adjustment procedures. The resulting low-lying dipole spectra in ${ }^{116,120} \mathrm{Sn}$ are compared with conventional RQTBA calculations. It has been found that the two-phonon correlations redistribute the fragmented strength as compared to the $2 \mathrm{q} \otimes$ phonon RQTBA so that the major fraction of the RQRPA pygmy mode is pushed above the neutron threshold and therefore mixed with the giant dipole resonance tail. The calculated low-energy fraction of the electric dipole strength agrees also very well with the available data for the tin isotopes and for the recently investigated neutron-rich nucleus 
${ }^{68} \mathrm{Ni}$. In general, the relativistic two-phonon model presented here provides a new quality of understanding of mode coupling mechanisms in nuclei. The method is based on Green's function techniques and can be widely applied also in other areas of quantum many-body physics.

Valuable discussions with H. Feldmeier and I. Mukha are gratefully acknowledged. This work was supported by the Hessian LOEWE initiative through the Helmholtz International Center for FAIR and the Russian Federal Education Agency Program, project 2.1.1/4779.

[1] N. Paar, D. Vretenar, E. Khan, G. Coló, Rep. Prog. Phys. 70, 691 (2007).

[2] N. Ryezaeva et al., Phys. Rev. Lett. 89, 272502 (2002).

[3] A. Zilges et al., Phys. Lett. B542, 43 (2002).

[4] D. Savran et al., Phys. Rev. Lett. 97, 172502 (2006).

[5] R. Schwengner et al., Phys. Rev. C 76, 034321 (2007).

[6] R. Schwengner et al., Phys. Rev. C 78, 064314 (2008).

[7] D. Savran et al., Phys. Rev. Lett. 100, 232501 (2008).

[8] P. Adrich et al., Phys. Rev. Lett. 95, 132501 (2005).

[9] A. Klimkiewicz et al., Phys. Rev. C 76, 051603(R) (2007).

[10] O. Wieland et al., Phys. Rev. Lett. 102, 092502 (2009).

[11] N. Paar, P. Ring, T. Nikšić, and D. Vretenar, Phys. Rev. C 67, 034312 (2003).

[12] V. Tselyaev et al., Phys. Rev. C 75, 014315 (2007).

[13] N. Tsoneva and H. Lenske, Phys. Rev. C 77, 024321 (2008).

[14] J. Terasaki and J. Engel, Phys. Rev. C 74, 044301 (2006).

[15] E. Litvinova, P. Ring, and V. Tselyaev, Phys. Rev. C 78, 014312 (2008).

[16] E. Litvinova, P. Ring, V. Tselyaev, and K. Langanke, Phys. Rev. C 79, 054312 (2009).

[17] P.O. Lipas, Nucl. Phys. 82, 91 (1971).

[18] P. Vogel and L. Kocbach, Nucl. Phys. A176, 33 (1971).

[19] J. Bryssinck et al., Phys. Rev. C 59, 1930 (1999).

[20] M. Wilhelm, E. Radermacher, A. Zilges and P. von Brentano, Phys. Rev. C 54, R449 (1996).

[21] I. Pysmenetska et al., Phys. Rev. C 73, 017302 (2006). 
[22] B. Özel et al., Nucl. Phys. A 788, 385c (2007).

[23] V. I. Tselyaev, Phys. Rev. C 75, 024306 (2007).

[24] G. A. Lalazissis, J. König, and P. Ring, Phys. Rev. C 55, 540 (1997).

[25] K. Govaert et al., Phys. Rev. C 57, 2229 (1998).

[26] N. Tsoneva, H. Lenske, Ch. Stoyanov, Phys. Lett. B 586, 213 (2004).

[27] B. Özel, Ph. D. thesis, Cukurova University (2008); private communication. 